\title{
Incorporating lung cancer screening education into tobacco cessation group counseling
}

\author{
Dan J. Raz', Mohamed H. Ismail2, Virginia Sun ${ }^{3}$, Stacy Park', Angel C. Alem ${ }^{4}$ Eric C. Haupt ${ }^{4}$, Michael K. Gould ${ }^{4}$
}

\begin{abstract}
INTRODUCTION Lung cancer screening (LCS) with low-dose computed tomography (LDCT) reduces lung cancer mortality, yet few people who are eligible for LCS get screened. In the present study, we studied the feasibility of tobacco cessation counselors to inform about LCS during tobacco cessation group counseling.

METHODS Four tobacco cessation counselors at two different facilities offering group tobacco cessation counseling were trained to administer an educational intervention about LCS. The intervention was administered to 25 participants during May 2019 who completed surveys that assessed how much the information provided helped with understanding various aspects of lung cancer screening including benefits, risks, eligibility criteria, and insurance coverage. The intervention also provided information on how to learn more about LCS and assessed the acceptability of the information.

RESULTS The median score for understanding of all components of the intervention was 5 ('completely understand'). Most participants (92\%) felt that the information provided about LCS was the right amount. Most participants (72\%) were aged 55-80 years, the age range for LCS eligibility. Four participants (16\%) reported undergoing LCS in the past. When we reanalyzed the subset of participants who reported no prior LCS, the results of surveys were similar.

CONCLUSIONS Our findings suggest that it is feasible to train tobacco cessation counselors to educate smokers, attending group tobacco cessation counseling classes, also about LCS. The education provided in this study was both understood and well received by the large majority of smokers surveyed. Further study is needed to understand the effect of LCS education on utilization of LDCT among smokers enrolled in tobacco cessation counseling.
\end{abstract}

\section{AFFILIATION}

1 Division of Thoracic Surgery, City of Hope, Duarte, United States 2 Department of Preventive Medicine, Kaiser Permanente, Riverside, United States 3 Department of Population Sciences, City of Hope, Duarte, United States

4 Department of Research and Evaluation, Kaiser Permanente Southern California, Pasadena, United States

CORRESPONDENCE TO Dan J. Raz. Division of Thoracic Surgery, City of Hope, $1500 \mathrm{E}$. Duarte Rd, Duarte, CA 91010, United States. E-mail: draz@coh.org

\section{KEYWORDS}

smoking cessation, lung cancer screening, cessation education

Received: 18 October 2019 Revised: 6 December 2019 Accepted: 9 December 2019

\section{INTRODUCTION}

Close to $30 \%$ of all cancer deaths are due to lung cancer ${ }^{1}$. Lung cancer screening (LCS) with low-dose computed tomography (LDCT) reduces lung cancer mortality ${ }^{2,3}$. The National Comprehensive Cancer Network (NCCN) and US Preventive Services Task Force (USPSTF) guidelines recommend LCS in high-risk patients, as do a number of other professional organizations ${ }^{4,5}$. The USPSTF recommends screening current and former smokers aged $55-80$ years who have smoked $\geq 30$ packyears, and who quit within 15 years if a former smoker.
Although there is limited information on the proportion of high-risk people who undergo LCS, Pinsky and Berg6 estimated, using National Lung Screening Trial (NLST) criteria, that $6.2 \%$ of adults over the age of 40 years would be eligible for screening. ${ }^{6}$. Yet, the number of patients screened at most LCS centers is anecdotally very low. We recently reported that only $8 \%$ of primary care physicians reported referring most or all high-risk patients to an LCS center during the prior 12 months ${ }^{7}$. A number of barriers to utilization of LCS in high-risk patients exist. 
We recently conducted a survey of primary care physicians (PCPs) and identified that concerns regarding the effectiveness of LDCT to prevent death from lung cancer, potential risks of LDCT, and costs of LDCT were of greatest concern to PCPs ${ }^{7}$. We also identified that few PCPs have a mechanism to identify patients eligible for screening in an automated fashion because of the way that tobacco exposure is documented in the electronic medical record (EMR). We reported that capturing a detailed tobacco exposure history in the EMR improved utilization of LCS and tobacco cessation services ${ }^{8}$. Novel systems approaches are needed to overcome barriers and improve utilization of LCS.

In addition to the life-saving potential of early detection of lung cancer, LCS offers additional value by serving as a point-of-entry into tobacco cessation counseling for current smokers. Integration of tobacco cessation into LCS programs is mandated by the Centers for Medicare Services (CMS). Although a recent small interview study reported that a large proportion of current smokers expressed that LCS might lower their motivation for tobacco cessation ${ }^{9}$, data from the National Lung Screening Trial and from the International Early Lung Action Program have shown that LCS stimulates tobacco cessation and that cessation rates are highest among patients with abnormal screening test results ${ }^{10-12}$.

A far larger number of current smokers seek out tobacco cessation services, such as telephone quitlines, tobacco cessation groups, or individual counseling, compared with the number of smokers screened with LDCT. For example, Davis et al. ${ }^{13}$ reported that 363656 calls were made to state quitlines over a 12 -week period in 2012 . Yet, most tobacco cessation services offer no education on LDCT. Moreover, the vast majority of smokers who are eligible for LCS and seek out tobacco cessation services do not know about the potential benefits of $\mathrm{LDCT}^{14}$. Education by tobacco cessation counselors on the benefits of LDCT and where to be screened, may be an important way to improve adherence to LCS guidelines. Questions regarding the effectiveness of this approach warrant investigation. These include the feasibility of LCS education in a variety of tobacco cessation counseling settings (oneon-one counseling, group counseling, telephone quitlines, and online courses) and the type of education that is most effective, but still within the scope of practice of the counselor.

In this study, we explored the feasibility of training tobacco cessation counselors in a large health system to educate smokers, attending group tobacco cessation classes, on lung cancer screening within a large health system.

\section{METHODS}

Current smokers who attended at least one tobacco cessation group session, part of a four-session course entitled 'Freedom from Tobacco,' at one of two participating Kaiser Permanente medical centers in Southern California, were eligible to participate during May 2019. A total of 25 volunteer participants completed surveys at the end of the class after distribution of the survey by tobacco cessation counselors. No class participant declined to complete a survey. Anonymous surveys were completed on paper forms and completed surveys were collected and mailed to City of Hope for analysis by study staff. Participants were not compensated for survey completion. This study was reviewed and approved by the Kaiser Permanente Institutional Review Board. A waiver of informed consent was obtained as protected health information was not collected as part of the survey.

\section{Training of tobacco cessation counselors}

Four tobacco cessation counselors at two sites, where group tobacco cessation counseling was offered, administered the study educational intervention. The training backgrounds of the counselors are summarized in Table 1 . The counselors were trained on lung cancer screening by the principal investigator by reviewing detailed information about the data supporting lung cancer screening, and details about eligibility requirements, risks, harms, and logistics including how patients can get screened and insurance

\section{Table 1. Tobacco cessation counselors}

$\begin{array}{llc}\text { Job tille } & \begin{array}{c}\text { Educational background } \\ \text { Participants } \\ \text { traninced in (\%) }\end{array} \\ \text { Health Educator } & \begin{array}{l}\text { Master's in Public Health and } \\ \text { Health Administration }\end{array} & 9(36) \\ \text { Health Educator } & \text { Master's in Public Health } & 9(36) \\ \text { Health Educator } & \text { Registered Dietician } & 3(12) \\ \text { Lifestyle Educator } & \text { Bachelor's } & 4(16)\end{array}$


coverage. The counselors were then provided with a script in outline form on the content to deliver during tobacco cessation classes. In addition, printed patient education material was reviewed with cessation counselors and they were instructed to distribute this material to participants receiving the educational intervention for reinforcement. Upon completion of the pilot program, the cessation counselors were contacted regarding their experience and to provide feedback to the study team.

\section{Survey items and measures}

The survey was structured into five parts, and took approximately 5 minutes to complete. First, participants were asked how much the information provided helped with understanding various aspects of lung cancer screening including benefits, risks from how screening is done, eligibility criteria, insurance coverage, and who to contact for more information about LCS using a Lickert scale of 1-5. Participants were then asked about whether the amount of information provided was the right amount and how satisfied they were with answers provided by cessation counselors to any questions asked. An open-ended question soliciting comments about the intervention was also included. Finally, sociodemographic characteristics including age, race, income, education, and smoking history were collected.

\section{Data analysis and statistics}

Descriptive statistics including counts and percentages were used to summarize participants' sociodemographic information, current smoking status, and opinions about the amount of information provided about lung cancer screening. Responses regarding understanding of the components of LCS education were summarized as median of the Lickert scale response. For questions pertaining to satisfaction with answers to questions asked, results were summarized after being grouped into agree/strongly agree and disagree/strongly disagree categories. Any differences between the study sample and the combined totals in Tables 2 and 3 are due to missing data.

\section{RESULTS}

We included 25 smokers in the analysis. Their sociodemographics are summarized in Table 2. Most participants $(72 \%)$ were aged $55-80$ years, the age
Table 2. Sociodemographic characteristics and smoking history of study participants

\begin{tabular}{lc} 
Characteristics & n ( $\%)$ \\
Male & $15(60)$ \\
Age (years) & \\
$<45$ & $3(12)$ \\
$45-54$ & $4(16)$ \\
$55-65$ & $12(48)$ \\
$65-75$ & $6(24)$ \\
$>76$ & 0 \\
Race ethnicity & \\
Non-Hispanic White & $11(44)$ \\
Black & $7(28)$ \\
Hispanic/Latino & $4(16)$ \\
Asian/Pacific Islander & 0 \\
Other/Multi-Racial & $3(12)$ \\
Education level & \\
Some high school or less & $1(4)$ \\
High school & $7(28)$ \\
Some college & $11(44)$ \\
College degree & $4(16)$ \\
Some post-college education & 0 \\
Post-graduate degree & $2(8)$ \\
Pack-years smoking & \\
median (IQR) & $40(15-53)$ \\
& \\
\hline &
\end{tabular}

Table 3. Response to survey questions

\begin{tabular}{|c|c|}
\hline $\begin{array}{l}\text { Ilow much did the information about LCS } \\
\text { help with your understanding of: }\end{array}$ & $\begin{array}{c}\text { Vedian (IQR, } \\
\text { score range } \\
1-5)\end{array}$ \\
\hline Benefits of LCS & $5(5-5)$ \\
\hline How LCS is done & $5(5-5)$ \\
\hline Who is eligible for LCS & $5(5-5)$ \\
\hline Risks of LCS & $5(5-5)$ \\
\hline LCS coverage by insurance & $5(5-5)$ \\
\hline Who to contact to learn more about LCS & $5(5-5)$ \\
\hline Was the information provided about LCS: & n $(\%)$ \\
\hline Too little & $2(8)$ \\
\hline The right amount & $23(92)$ \\
\hline Too much & 0 \\
\hline
\end{tabular}

How satisfied with the answers given to in (\%o) questions asked albout LCS:

Very or somewhat satisfied

$22(88)$

Very or somewhat dissatisfied

$1(4)$

No questions were asked 
range for LCS eligibility. The median pack-year history of smoking was 40 , and $64 \%$ of participants had a smoking history of 30 pack-years or greater. The population was racially/ethnically diverse. Participants had varied educational backgrounds, with $32 \%$ of participants completing some or all of high school as their highest level of education. Four participants (16\%) reported that they had previously undergone lung cancer screening.

Overall, the intervention was well received. The median score for the understanding of all components of the intervention was 5 ('completely understand'). Most participants (92\%) felt that the information provided about LCS was the right amount, while the remaining few participants felt that there was too little information. In addition, most participants $(88 \%)$ were satisfied with the answers that the cessation counselors provided to questions asked during the session. No survey responder provided any specific comments about their experience or suggestions for improvement on the survey in response to the open-ended questions asked about the educational intervention.

We re-analyzed the study data after restricting our analysis to 21 participants who responded that they had never undergone lung cancer screening. Results were similar to the over group with regards to median score for understanding of the components of the intervention (5), the amount of information provided during the intervention $(90 \%$ reported the right amount of information provided), and satisfaction with answers to questions asked (86\% very or somewhat satisfied).

\section{DISCUSSION}

The study suggests that it is feasible to train tobacco cessation counselors to educate smokers, attending group tobacco cessation counseling classes, about LCS. Education provided in this study was both understood and well received by the large majority of smokers surveyed. This is the first study to our knowledge investigating the feasibility of training tobacco cessation counselors to educate smokers about LCS. There is considerable effort to incorporate tobacco cessation into lung cancer screening programs ${ }^{12,15}$. In the United States, tobacco cessation services are required for sites in order for reimbursement to be given by the Center for Medicare and Medicaid
Services (GMS). A number of studies have been published evaluating different modalities of tobacco cessation counseling for patients undergoing LCS $^{16-20}$.

While use of LCS as a teachable moment for tobacco cessation is important, far less has been known about use of tobacco cessation as a teachable moment for LCS. A far larger number of smokers seek out tobacco cessation services than $\mathrm{LCS}^{7,13}$. While educating smokers on LCS, which is recommended by the U.S. Preventive Services Task Force and the American Cancer Society, may be wellintentioned, there are several issues that should be clarified. Again, our study shows that it is feasible for tobacco cessation counselors to administer education regarding lung cancer screening. While the U.S. Public Health Service recommends tobacco cessation counseling training for all healthcare professionals, the educational and training background of cessation counselors providing most cessation services can vary $^{21}$. There are other important issues that need additional investigation, including what kind of LCS education and how much information should be administered. LCS education can be given in person, by telephone, or through video or online content. The format of education and length of the educational intervention may also depend on the type of cessation counseling that is administered, i.e. one-on-one, group counseling, phone counseling, or online counseling. Most importantly, the effect of the educational intervention on utilization of LCS has not been determined. It is also unclear whether such educational intervention has an effect on abstinence from tobacco. Several studies have shown that participating in LCS does not lead to increased smoking rates, and may lead to increased smoking cessation rates ${ }^{11,22}$, but it is not known whether LCS education affects tobacco cessation rates in those seeking tobacco cessation services.

\section{Limitations}

Our study has several limitations. First, it is a relatively small study at two sites administering group tobacco cessation classes within a single healthcare system. These results may differ in other tobacco cessation settings, such as phone counseling. Although the number of participants was small, it was fairly diverse with respect to race/ethnicity and educational background, which should make this generalizable to 
most US populations. In this study, we explored the feasibility of a brief LCS educational intervention that included printed literature for the participant to take home. More extensive educational interventions may result in different acceptance than what we found. Finally, this study was designed only to test feasibility of the intervention in this patient population. Additional studies are needed on the effect of an LCS educational intervention that is delivered by tobacco cessation counselors, at the time of tobacco cessation counseling, on LCS utilization and tobacco cessation rates.

\section{CONCLUSIONS}

We found that it is feasible to train tobacco cessation counselors to deliver an educational intervention, and tobacco cessation counseling may be an opportune time to educate smokers about the benefits of LCS.

\section{REFERENCES}

1. American Cancer Society. Cancer Facts \& Figures 2016. https://www.cancer.org/research/cancer-facts-statistics/ all-cancer-facts-figures/cancer-facts-figures-2016.html. Accessed October 18, 2019.

2. The International Early Lung Cancer Action Program Investigators. Survival of patients with stage I lung cancer detected on CT screening. N Engl J Med. 2006;355(17):1763-1771. doi:10.1056/nejmoa060476

3. National Lung Screening Trial Research Team, Aberle DR, Adams AM, et al. Reduced lung-cancer mortality with lowdose computed tomographic screening. N Engl J Med. 2011;365(5):395-409. doi:10.1056/nejmoa1102873

4. Moyer VA, U. S. Preventive Services Task Force. Screening for lung cancer: U.S. Preventive Services Task Force recommendation statement. Ann Intern Med. 2014;160(5):330-338. doi:10.7326/m13-2771

5. Wood DE, Kazerooni EA, Baum SL, et al. Lung Cancer Screening, Version 3.2018, NCCN Clinical Practice Guidelines in Oncology. J Natl Compr Canc Netw. 2018;16(4):412-441. doi:10.6004/jnccn.2018.0020

6. Pinsky PF, Berg CD. Applying the National Lung Screening Trial eligibility criteria to the US population: what percent of the population and of incident lung cancers would be covered? J Med Screen. 2012;19(3):154-156. doi:10.1258/jms.2012.012010

7. Raz DJ, Wu GX, Consunji M, et al. Perceptions and utilization of lung cancer screening among primary care physicians. J Thorac Oncol. 2016;11(11):1856-1862. doi:10.1016/j.jtho.2016.06.010

8. Raz DJ, Dunham R, Tiep B, et al. Augmented meaningful use criteria to identify patients eligible for lung cancer screening. Ann Thorac Surg. 2014;98(3):996-1002. doi:10.1016/j.athoracsur.2014.04.105

9. Zeliadt SB, Heffner JL, Sayre G, et al. Attitudes and Perceptions About Smoking Cessation in the Context of Lung Cancer Screening. JAMA Intern Med. 2015;175(9):15301537. doi:10.1001/jamainternmed.2015.3558

10. Ostroff JS, Buckshee N, Mancuso CA, et al. Smoking cessation following CT screening for early detection of lung cancer. Prev Med. 2001;33(6):613-621. doi:10.1006/pmed.2001.0935

11. Tammemagi MC, Berg CD, Riley TL, et al. Impact of lung cancer screening results on smoking cessation. J Natl Cancer Inst. 2014;106(6):dju084. doi:10.1093/jnci/dju084

12. Taylor KL, Cox LS, Zincke N, et al. Lung cancer screening as a teachable moment for smoking cessation. Lung Cancer. 2007;56(1):125-134. doi:10.1016/j.lungcan.2006.11.015

13. Davis KC, Alexander RLJ, Shafer P, et al. The DoseResponse Relationship Between Tobacco Education Advertising and Calls to Quitlines in the United States, March-June, 2012. Prev Chronic Dis. 2015;12:E191. doi:10.5888/pcd12.150157

14. Raz DJ, Wu G, Nelson RA, et al. Perceptions and Utilization of Lung Cancer Screening Among Smokers Enrolled in a Tobacco Cessation Program. Clin Lung Cancer. 2019;20(1):e115-e122. doi:10.1016/j.cllc.2018.09.013

15. Steliga MA, Yang P. Integration of smoking cessation and lung cancer screening. Transl Lung Cancer Res. 2019;8(Suppl 1):S88-S94. doi:10.21037/tlcr.2019.04.02

16. Cadham CJ, Jayasekera JC, Advani SM, et al. Smoking cessation interventions for potential use in the lung cancer screening setting: A systematic review and meta-analysis. Lung Cancer. 2019;135:205-216. doi:10.1016/j.lungcan.2019.06.024

17. Tremblay A, Taghizadeh N, Huang J, et al. A Randomized Controlled Study of Integrated Smoking Cessation in a Lung Cancer Screening Program. J Thorac Oncol. 2019;14(9):1528-1537. doi:10.1016/j.jtho.2019.04.024

18. Meltzer LR, Unrod M, Simmons VN, et al. Capitalizing on a teachable moment: Development of a targeted selfhelp smoking cessation intervention for patients receiving lung cancer screening. Lung Cancer. 2019;130:121-127. doi:10.1016/j.lungcan.2019.02.014

19. Iaccarino JM, Duran C, Slatore CG, et al. Combining smoking cessation interventions with LDCT lung cancer screening: A systematic review. Prev Med. 2019;121:2432. doi:10.1016/j.ypmed.2019.02.016

20. Joseph AM, Rothman AJ, Almirall D, et al. Lung Cancer Screening and Smoking Cessation Clinical Trials. SCALE (Smoking Cessation within the Context of Lung Cancer Screening) Collaboration. Am J Respir Crit Care Med. 2018;197(2):172-182. doi:10.1164/rccm.201705-0909ci

21. Clinical Practice Guideline Treating Tobacco Use and Dependence 2008 Update Panel, Liaisons, and Staff. A clinical practice guideline for treating tobacco use and dependence: 2008 update. A U.S. Public Health Service report. Am J Prev Med. 2008;35(2):158-176. 
doi:10.1016/j.amepre.2008.04.009

22. van der Aalst CM, van Klaveren RJ, van den Bergh KA, et al. The impact of a lung cancer computed tomography screening result on smoking abstinence. Eur Respir J. 2011;37(6):1466-1473. doi:10.1183/09031936.50035410

ACKNOWLEDGEMENTS

We thank the tobacco cessation counselors who participated in this study: Catherine Chanady, Latyce Moyo, Sarah Delcourt, Andile Ndlela and Katie Wical.

CONFLICTS OF INTEREST

The authors have each completed and submitted an ICMJE form for disclosure of potential conflicts of interest. The authors declare that they have no competing interests, financial or otherwise, related to the current work. D. J. Raz reports financial activities outside the submitted word.

FUNDING

This study was funded through a grant from the Lung Cancer Research Foundation (DR).

PROVENANCE AND PEER REVIEW

Not commissioned; externally peer reviewed. 